Diabetologe 2020 $\cdot 16: 4-5$

https://doi.org/10.1007/s11428-019-00527-4

(c) Springer Medizin Verlag GmbH, ein Teil von Springer Nature 2019

Wenn Patienten mit Diabetes heutzutage zu einer Visite in die Praxis ihres behandelnden Diabetologen kommen, geht es vielfach nicht vorrangig um eine Optimierung der Therapie mit antidiabetischen Medikamenten, sondern zunächst kommunizieren der Patient und der behandelnde Arzt/die Mitglieder des Diabetesteams über die Güte der Glukosekontrolle in den letzten Wochen und Monaten. Dazu werden die Daten eines Systems zum kontinuierlichen Glukosemonitoring ausgelesen und besprochen. Hinzu kommen in vielen Fällen die Daten von einem Smartpen oder einer konventionellen Insulinpumpe bzw. einer Patch-Pumpe. Möglicherweise hat der Patient noch zusätzliche Informationen auf seinem Smartphone zu seinem Bewegungsverhalten, seinen Essensgewohnheiten usw., die von ihm selbst oder dem Phone/Wearable erfasst werden. Die Gesamtheit aller dieser Daten kann vom Behandler kaum noch erfasst und interpretiert werden. Liegen die Daten an einer Stelle in der Praxis oder in der Cloud vor, ist durch den Einsatz smarter Algorithmen eine unterstützende Analyse möglich, die konkrete Hinweise liefert. All dies ist keine Zukunftsmusik, sondern jetzt schon verfügbar und wird spätestens im nächsten Jahr (2020) Realität werden.

Diese Gedanken sind der Grund, warum wir Menschen, die konkret mit Patienten arbeiten, baten, aus ihrem jeweiligen Blickwinkel ihre Gedanken, Themen und Fragen zum Einsatz von Diabetestechnologie (DT) im Alltag darzustellen. Es geht uns nicht darum, eine bunte neue Welt schön $\mathrm{zu}$ reden, sondern darzulegen, was möglich ist, was nicht geht und was es gilt, zu tun! An der Betreuung von Menschen mit Diabetes sind eine Reihe von Menschen und

\author{
Matthias Kaltheuner' ${ }^{1}$ Lutz Heinemann ${ }^{2}$ \\ 'Diabetologikum Leverkusen, Leverkusen, Deutschland \\ ${ }^{2}$ Science Consulting in Diabetes $\mathrm{GmbH}$, Neuss, Deutschland
}

\title{
Diabetestechnologie in der Praxis
}

Organisationen beteiligt, daher kommt zunächst ein Diabetologe zu Wort, der über seine Erfahrungen mit DT in einer Diabetesschwerpunktpraxis berichtet. Eine zentrale Rolle bei der Nutzung von DT haben die Diabetesberater. Bisher hatte DT in deren Ausbildung eine eher untergeordnete Bedeutung, dies ändert sich aktuell. Schulung wird in Zukunft anders erfolgen als in den letzten 30 Jahren. In den Praxen erfordert der Einsatz von DT (und der Digitalisierung der Praxen) erhebliche Änderungen in den Prozessen und Abläufen, dies bedingt eine Bereitschaft aller Beteiligten, sich solchen Anforderungen zu stellen.

Begleitend und auf übergeordneter Ebene sind sowohl die Fachgesellschaft als auch die Arbeitsgemeinschaft der DDG (Deutsche Diabetes Gesellschaft), die sich mit DT beschäftigt, an vielen Stellen aktiv, sei es durch Stellungnahmen und Positionspapiere dazu, sei es durch die Organisation von und die Beteiligung an Fortbildungen, sei es durch Kommunikation mit Landes- und Bundesbehörden im Gesundheitssektor.

\section{》) Implementierung von \\ Diabetestechnologie in die Praxis ist eine aktuelle, keine künftige Aufgabe}

Haupthindernisse bei der Implementierung und Umsetzung von DT in der Praxis sind die als komplex wahrgenommenen IT-Strukturen (IT: Informationstechnik), Kostenfragen sowie juristische Aspekte. Viele Kollegen sind aus solchen praktisch hochrelevanten und teilweise recht komplexen Gründen beim Thema DT zurückhaltend. Durch Intensivierung der Kommunikation mit geeigneten Ju- risten sind viele Probleme allerdings gut lösbar.

Aus Sicht der Patienten hat sich die Interaktion mit dem Diabetesteam erheblich geändert, und dies wird auch weiterhin der Fall sein. Der Patient kann und muss nicht mehr für jedes Rezept in die Praxis kommen, er kann mit dem Behandler über andere Kanäle kommunizieren, eine Fernbehandlung ist keine Science-Fiction mehr, sondern wird Realität. Patienten kommen heute vielfach wesentlich informierter in die Praxis, nicht nur haben sie sich im Internet zu neuen Entwicklungen und Optionen informiert, sie analysieren selbst ihre Daten und haben konkrete Fragen zu ihrer Therapie. Diabetestechnologie, die hilft, die Stoffwechsellage leichter und besser $\mathrm{zu}$ steuern, bewirkt eine Verbesserung der Lebensqualität, also ein besseres Leben für die chronisch Kranken mit Diabetes. So ermöglicht die kontinuierliche Glukosespiegelmessung für viele Patienten erstmals ein Selbstmanagement, da ihnen jetzt die nötigen Informationen zur Verfügung stehen. Gefühle von Überforderung und Unfähigkeit können überwunden werden. Beispiele von einzelnen Patienten verdeutlichen, was die Nutzung der neuen Technologien bewirken kann.

Der Einsatz von DT führt zu einer gewissen Steigerung der Kosten für die Krankenversicherungen, wenn diese ihren Versorgungsauftrag ernst nehmen. In diesem Fall wird nicht nur die Betreuung der Patienten verbessert (was auf längere Sicht zu einer Kostenreduktion führen sollte), sondern es geht den Betroffenen real besser, sie haben z. B. weniger akute Glukosespiegelentgleisungen. Wenn die Anzahl schwerer Hypoglykämien durch den Einsatz von DT reduziert wird, hat dies neben Kostenfragen erhebliche positive psychologische Auswirkungen, nicht 
nur für die Patienten selbst, sondern auch für deren Angehörige. Der Transfer von Daten ermöglicht es Eltern von Kindern mit Diabetes, deren Glukosespiegelkontrolle während der Nacht im Blick zu haben, aber auch während der Zeit, die diese im Kindergarten oder der Schule verbringen. Im gleichen Sinne erleichtert und ermöglicht DT Patienten mit Diabetes in vielen Fällen, Berufe zu wählen und auszuführen, die ihnen früher quasi verboten waren. Dies geht dahin, dass Diabetespatienten heute auch Piloten von Verkehrsflugzeugen sein können.

Die Hersteller von Medizinprodukten, die bei der Diagnose und Therapie von Diabetes genutzt werden, sind heute Partner der Behandler und der Patienten. Das angesprochene Zusammenwachsen aller Bereiche, die bei der Betreuung von Patienten zusammenarbeiten, verlangt auch von den Industriepartnern Umstellungen und Änderungen bei deren Businessmodellen und Herangehensweisen.

Insgesamt veränderten diese Entwicklungen die Diabetestherapie in den letzten Jahren - und noch mehr in den Jahren, die vor uns liegen - essenziell. Für die Patienten und die Behandlungseffizienz und -sicherheit hat dies klare Vorteile, es gilt, alle Strukturen, die in die Behandlung involviert sind, entsprechend mit einzubeziehen.
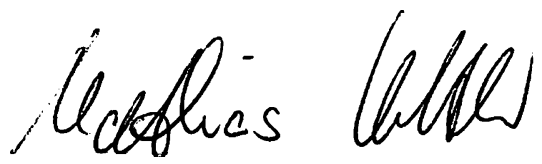

Dr. Matthias Kaltheuner
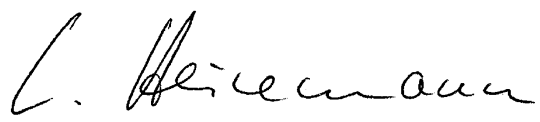

Prof. Dr. Lutz Heinemann

\section{Korrespondenzadresse}

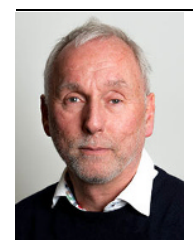

Dr. Matthias Kaltheuner

Diabetologikum Leverkusen

Leverkusen, Deutschland

m.kaltheuner@web.de

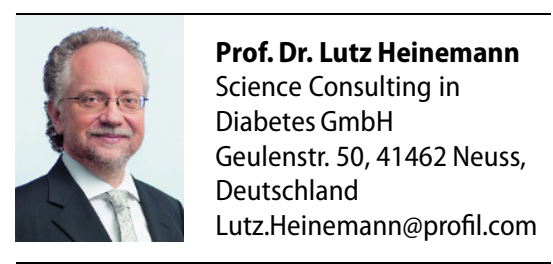

Interessenkonflikt. L. Heinemann ist Anteilseigner bei dem Profil Institut für Stoffwechselforschung $\mathrm{GmbH}$ in Neuss und bei ProSciento in San Diego, USA. Des Weiteren ist er als Berater für eine Reihe von Firmen, die neue diagnostische und therapeutische Optionen für die Diabetestherapie entwickeln, tätig. M. Kaltheuner gibt an, dass kein Interessenkonflikt besteht.

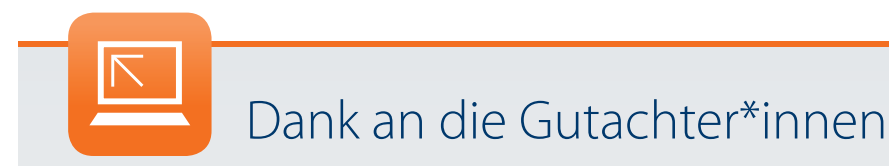

Für die Qualität und Objektivität der Beiträge sind neben den engagierten Autorinnen und Autoren auch die vielen qualifizierten Gutachterinnen und Gutachter maßgeblich, die im Rahmen des Peer-Review-Prozesses die Manuskripte inhaltlich-wissenschaftlich prüfen und Empfehlungen zur konkreten Verbesserung äußern. Allen Gutachterinnen und Gutachtern, die im vergangenen Jahr Manuskripte für diese Zeitschrift begutachtet haben, danken wir herzlich für die konstruktive und gewissenhafte Arbeit.

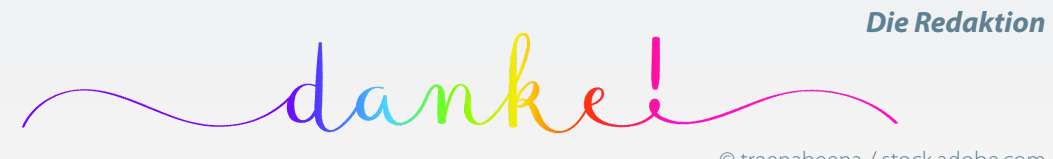

(ㄷ) treenabeena / stock.adobe.com 